\title{
COMPRENSIÓN DE LA CALIDAD DE VIDA EN PERSONAS MAYORES SEMIVALENTES EN CHILE ${ }^{1}$
}

COMPREHENDING QUALITY OF LIFE IN SEMI-ABLED OLDER PEOPLE IN CHILE

Recibido: 24 de Marzo del 2011 | Aceptado: 30 de Agosto del 2011

\author{
PAULINA OSORIO PARRAGUEZ ${ }_{2} ;$ MARIA JOSE TORREJON $_{3}$; CYNTHIA MEERSOHN $_{4} ;$ MARIA SOL ANIGSTEIN $_{5}$ \\ (UNIVERSIDAD DE CHILE, Santiago de Chile, CHILE)
}

\begin{abstract}
RESUMEN
Este artículo presenta resultados de un estudio cuyo objetivo es conocer y analizar las percepciones, creencias y evaluaciones sobre su calidad de vida en hombres y mujeres mayores semivalentes. Se trata de una investigación cualitativa que incluyó entrevistas en profundidad a personas mayores semivalentes mayores de 60 años de la Región Metropolitana, analizadas con asistencia del software Atlas ti 5.0. Los resultados muestran que las dimensiones que más afectan la percepción de calidad de vida de las personas mayores semivalentes son la economía, la familia y la salud, observándose diferencias por género en su jerarquía y manifestación. El análisis de los datos sugiere que las dimensiones de la calidad de vida no son absolutas, por lo que se propone un diagrama interpretativo que entiende a las dimensiones como condiciones y entornos, y que incorpora en su comprensión las experiencias y significados individuales y sociales, asumiendo que la calidad de vida, no es o positiva 0 negativa, buena 0 mala, sino que su percepción dependerá de la condición desde la que se evalúe y del entorno en que se la experimente.

PALABRAS CLAVE: Calidad de vida, envejecimiento, personas semivalentes, autonomía/dependencia.
\end{abstract}

\section{ABSTRACT}

This article presents results of a study which objective is to identify and analyze the perceptions, beliefs and evaluations of the quality of life in semi-able older men and women. This is a qualitative research that includes indepth interviews to semi-able seniors over 60 years old in the Metropolitan Region. The analysis was executed with the assistance of Atlas ti 5.0 software. The results show that the dimensions that affected the perceived quality of life of semi-able older people were economy, family and health; gender differences were observed in their hierarchy and manifestation. The analysis suggests that the quality of life dimensions are not absolute. Thus, we propose an interpretative diagram that understands the dimensions as conditions and environments, and incorporates the experiences as well as individual understanding and social meanings, assuming that the quality of life is neither positive nor negative, good nor bad. Instead, its perception will depend on the condition from which is evaluated and the environment in which it is experienced.

KEY WORDS: Quality of life, aging, semi- abled people, autonomy/dependence.

\footnotetext{
1. Trabajo desarrollado en el marco de la investigación "Calidad de Vida y Adulto Mayor", financiada por la Vicerrectoría de Investigación y Desarrollo de la Universidad de Chile, y que se inscribe en el interior del Programa Domeyko en Salud- Envejecimiento (2007-2010).

2.Profesora Asociada, Departamento de Antropología, Universidad de Chile.Santiago, Chile. Email: posorio@uchile.cl

3. Antropóloga Social, Magíster en Antropología y Desarrollo, Investigadora Programa Domeyko Salud-Envejecimiento, Universidad de Chile. Santiago, Chile. Email: mitorrejon@gmail.com

4. Antropóloga Social, Magíster en Antropología y Desarrollo, Investigadora Programa Domeyko Salud-Envejecimiento, Universidad de Chile. Santiago, Chile. Email: cynthia.meersohn@gmail.com

5. Antropóloga Social, Magíster en Antropología y Desarrollo, Investigadora Programa Domeyko Salud-Envejecimiento, Universidad de Chile.

Santiago, Chile. Email: sol.anigstein@gmail.com.
} 


\section{INTRODUCCIÓN}

Desde la emergencia de los estudios sobre calidad de vida y su incorporación en las reflexiones disciplinarias, la sociedad ha incorporado nuevos valores y estilos de vida. El aumento de la población mayor y sus consecuencias en las distintas sociedades, ha popularizado el uso del concepto de calidad de vida vinculado a los adultos mayores. En este marco, resulta pertinente observar el concepto desde una perspectiva dinámica, que reconozca la complejidad de su conocimiento, definición y medición.

La población mundial está envejeciendo, las proyecciones muestran que para el 2050 , el $21,4 \%$ de la población tendrá 60 años o más. La sociedad chilena no es la excepción. CELADE (2006) estima que para ese mismo año, la población adulta mayor en nuestro país será más de un $28 \%$, lo que sumado al aumento de la esperanza de vida y la disminución de los menores de 15 años, significará el envejecimiento de la población asociado a fenómenos emergentes como por ejemplo, el aumento de las situaciones de dependencia y discapacidad. Según la encuesta Casen 12009 , la discapacidad en las personas aumenta junto con la edad, es decir, son discapacitadas el $19,1 \%$ de personas entre 61 y 70 años, el $24,8 \%$ de las personas entre 71 y 80 años, y el $42,8 \%$ entre las personas de más de 80 años.

En los adultos mayores, como en otros grupos de edad, ciertas situaciones de discapacidad pueden dar lugar a diferentes grados de dependencia. La dependencia es definida por la Organización Mundial de la Salud (2001) como "la restricción o ausencia de capacidad para realizar alguna actividad en la forma o dentro del margen que se

\footnotetext{
Encuesta de Caracterización Socioeconómica Nacional, realizada por el Ministerio de Planificación de Chile - MIDEPLAN, es una encuesta de hogares representativa a nivel nacional, regional, urbano y rural y comunal. La Encuesta CASEN permite a MIDEPLAN elaborar diagnósticos de la realidad socioeconómica del país, evaluar los programas sociales y sus resultados.
}

considera normal; entre las actividades observadas se encuentran las actividades de la vida diaria (asearse, vestirse, comer y beber y cuidar del propio bienestar), preparar la comida y cuidar de la vivienda, así como participar en la movilidad" (Berjano, 2005: 144).

Según datos del departamento de Estadísticas e Información del Ministerio de Salud (MINSAL), en Chile, el año 2006 de las 547.624 personas adultas mayores que se atienden en el Sistema Nacional de Salud (SNS), menos de la mitad eran autovalentes, y el resto se distribuía en autovalentes con riesgo (163.632), dependientes (138.525) y postrados (18.931). Las proporciones entre estas categorías iban variando conforme aumentaba la edad ${ }^{2}$, y de acuerdo con lo que se planteará en este artículo, las variaciones en la dependencia tendrían un correlato en la percepción que las personas adultas mayores tienen de su calidad de vida. Las personas mayores semivalentes, son aquellas que no son autovalentes, pero tampoco están postradas, por lo que en este artículo se lo homologará con lo que desde la geriatría es llamado fragilidad (Puts, Widershoven, Heldens, Lips y Deeg, 2007). La fragilidad es una situación, un equilibrio delicado, en el que se encuentran los individuos, que significa el riesgo de vivir eventos adversos como pueden ser caídas, discapacidad, dependencia, institucionalización o muerte (Puts et al., 2007).

La calidad de vida es un concepto que ha sido utilizado de diversas maneras, desde múltiples perspectivas y disciplinas, razón por la cual definirlo no es una tarea fácil. Si bien se populariza como concepto en los años sesenta (Hernández, 2004; Hidalgo, 2008) es en los setenta que los estudios sobre calidad de vida comienzan a cobrar importancia en el ámbito académico,

\footnotetext{
Las personas entre 65 y 79 años, son autovalentes en su mayoría, le siguen los autovalentes con riesgo, luego los dependientes. Lo que cambia entre las personas de 80 y más años, en donde de los 117.201 personas que se atendían en el SNS, 30.983 eran autovalentes, 26.383 eran autovalentes con riesgo y 50.295 eran dependientes.
} 
junto con una aparición cada vez más frecuente en artículos referidos al tema específicamente en la literatura biomédica, psicológica y de ciencias sociales en general.

Esta popularización del concepto tiene relación con algunos cambios operados en el mundo durante el siglo XX. Dichos cambios han implicado el traslado de la atención desde temas que se vinculan con el ganarle a la muerte, como el hambre y la pobreza, a temas relacionados con cómo vivir la vida, la felicidad y el bienestar durante toda la vida.

En la actualidad, a pesar de la prolífera producción de artículos e investigaciones sobre el tema desde diversas disciplinas, no ha sido posible establecer un consenso en relación a qué es y cómo se mide la calidad de vida (Aranibar, 2001).

Dijkers (2007) sostiene que hay muchas y diferentes conceptualizaciones sobre calidad de vida y argumenta que la popularidad del concepto lo llevó a ser adoptado por distintas disciplinas, y a ser utilizado desde la perspectiva propia de cada una de ellas, por ejemplo, la economía en base a la utilidad, la medicina en base a la salud, y así.

Una de las definiciones más utilizadas es la propuesta por la Organización Mundial de la Salud, en 1994, la que describe la calidad de vida como la "percepción del individuo de su posición en la vida en el contexto de la cultura y sistema de valores en los que vive $y$ en relación con sus objetivos, expectativas, estándares y preocupaciones" (Schwartzmann, 2003: 3).

Moons, Budts y De Geest (2006), trabajaron sobre la base de diversas conceptualizaciones de calidad de vida, tales como, vida normal, utilidad social, felicidad / afecto, satisfacción con la vida, satisfacción con dominios específicos, logro de objetivos personales, y capacidad natural, en base a lo cual sostienen que la mayoría de los artículos abordan la calidad de vida en términos de vida normal o como medición de utilidad.

Actualmente existe un cierto consenso sobre la necesidad de conceptualizar la calidad de vida desde una perspectiva multidimensional, considerando aspectos subjetivos de las personas, a la vez que aquellos que son objetivos y/o externos. Su enfoque necesita además ser dinámico y específico, siendo capaz de reconocer las particularidades de la población o grupo en cuestión e incorporar algunos elementos más genéricos 0 nomotéticos (Aranibar, 2001; Fernández, 1997; George, 2006; Lawton en Barros, Avendaño y Forttes, 2004).

Aranibar (2001) considera que al utilizar el concepto de calidad de vida en el estudio de la vejez y el envejecimiento, se deben tener en cuenta tres premisas claves. En primer lugar, la especificidad del concepto según su contexto de referencia, en este caso la vejez, que presenta diferencias con los otros grupos de edad en cuanto a aspectos $y$ factores que configuran $e$ intervienen la idea de calidad de vida. En segundo lugar, su multidimensionalidad, lo que significa que es preciso tomar en consideración tanto factores personales, como socioambientales los cuáles varían de acuerdo a la edad de las personas. $Y$ en tercer lugar, el concepto de calidad de vida en la vejez, al igual que en todas las edades, debe considerar los aspectos subjetivos como las apreciaciones, valoraciones $y$ evaluaciones sobre las distintas dimensiones de la calidad de vida, y los aspectos objetivos de las mismas.

A nuestro entender, la calidad de vida en las personas mayores incluye la percepción que de ella tienen los propios sujetos, la evaluación que realizan de su entorno social y ambiental, así como las alternativas propiciadas por los Estados, que llevarían a que sea posible percibir una buena calidad 
de vida en la vejez, y las condiciones particulares que han influido durante el proceso de envejecimiento individual, vale decir, su trayectoria de vida (Osorio, Torrejón y Vogel, 2008).

En la investigación sobre calidad de vida en personas mayores, uno de los grandes problemas que se identifican es lo inadecuado de algunos instrumentos de medición, ya que éstos han sido diseñados para medir la calidad de vida en jóvenes. Puts et al.(2007) sostienen que aquello que es indicado como importante para la calidad de vida varía con la edad. Para el caso de las personas mayores dichas particularidades se refieren, por ejemplo, al menor horizonte temporal para la proyección, un creciente interés por la integridad y la búsqueda del significado de la vida, la necesidad de elaborar la muerte de seres cercanos y queridos, la disminución e intensificación de relaciones sociales, el ser tratados por otros mayoritariamente en función de la edad opacando atributos de las personas. El envejecer, por lo tanto, lejos de ser un proceso únicamente individual, es un proceso colectivo con implicancias y condicionado por el entorno social en el que tiene lugar, lo que significa que el tiempo individual se halla integrado al tiempo social, estando cada vivencia de las personas significada por el paso del tiempo y por el momento en que fueron experimentadas (Osorio, 2006).

Un estudio realizado por Vera (2007), muestra que los adultos mayores identifican como relevante, para su calidad de vida, el tener paz y tranquilidad, mantener su espacio físico dentro de la vivienda, que se respeten sus bienes materiales, su libre expresión, la capacidad de decidir sobre su vida cotidiana y que su familia esté unida. En el caso de las personas mayores semivalentes, la calidad de vida estará cruzada por "la interacción entre la persona y el ambiente en el que vive" (Scahlock, 1999: 2), lo que actualmente se entiende como una perspectiva ecológica del fenómeno.

El abordaje de la calidad de vida en la vejez debe partir de la base de que las personas mayores tienen una larga trayectoria de vida, es decir, que han transitado por varias edades. Si bien esto significa que existen elementos y aspectos que intervienen en la calidad de vida comunes con otros grupos etáreos, existen especificidades en las personas mayores semivalentes que intervendrán en su percepción de calidad de vida y que serán descritas a continuación. En relación a ello, el objetivo de la investigación, es conocer y analizar las percepciones, creencias y evaluaciones sobre su calidad de vida en hombres y mujeres mayores semivalentes, cuyos resultados se presentan por medio de un diagrama dinámico que nos han permitido interpretarlos.

\section{METODOLOGÍA}

Dado que nuestra reflexión busca articular el entendimiento de la calidad de vida desde una perspectiva sociocultural amplia, que reconozca la importancia de las definiciones que comúnmente se han realizado, la investigación se centró en un grupo etáreo particular: hombres y mujeres semivalentes de 60 años y más residentes en la Región Metropolitana. Se trata de una investigación de carácter cualitativo, con un muestreo intencional, que no sigue las leyes del azar. Esto significa que el investigador es quien selecciona las unidades de la muestra. La definición de nuestra muestra siguió las orientaciones del muestreo teórico -una de las modalidades del muestreo intencional-, cuyo principio básico es generar teoría por medio de la colección, codificación y análisis de datos, en base a los cuales se va decidiendo los datos que se continuarán produciendo y en dónde encontrarlos. Por ello se profundizó en las diversas categorías que se deseaba estudiar y se produjo información relevante para el concepto 0 teoría que se estaba elaborando: calidad de 
vida en hombres y mujeres mayores semivalentes. El muestreo se dio por finalizado cuando se alcanzó el nivel de saturación, esto es, cuando ya no aparecían nuevos conceptos y categorías relevantes. Los criterios establecidos para la selección de la muestra se presentan en la Tabla №1

TABLA 1.

Criterios Muestrales

\begin{tabular}{cl}
\hline Sexo & Masculino \\
& Femenino \\
Edad & Tramo 1:60 a 75 años \\
Nivel & Tramo 2: 76 y más \\
Alto \\
Socioeconómico & Medio \\
(NSE) & Bajo \\
\hline
\end{tabular}

De acuerdo a estos criterios y a la lógica de saturación se realizaron diez entrevistas en profundidad. "Por entrevistas cualitativas en profundidad entendemos reiterados encuentros cara a cara entre el investigador y los informantes, encuentros éstos dirigidos hacia la comprensión de las perspectivas que tienen los informantes respecto de sus vidas, experiencias o situaciones, tal como las expresan con sus propias palabras" (Taylor y Bogdan, 1987:101).

El análisis de los datos está inspirado en el eje analítico de la Grounded Theory, que plantea que la teoría emerge de la selección de información, es decir, no se crean categorías de análisis a priori, sino que estas surgen desde el mismo análisis textual. Cuando ya no emergen nuevos conceptos, se ha llegado al punto de saturación. El análisis estuvo asistido por el programa Atlas.ti 5.0. Luego, las categorías fueron analizadas de forma independiente para hombres y mujeres, con el fin de relevar las diferencias de género en la percepción de la calidad de vida. Se revisaron las frecuencias de cada categoría y su contenido específico por género y finalmente se hizo un análisis comparativo que permitió la emergencia de componentes de percepción diferenciados y categorías estructurales unificadoras, sobre las cuáles se elaboró un diagrama interpretativo para la comprensión de la calidad de vida, que revisaremos en la discusión de este artículo.

\section{RESULTADOS}

Para analizar los discursos referidos a la calidad de vida de hombres y mujeres semivalentes se han identificado una serie de dimensiones. Si bien las dimensiones son muy similares para ambos géneros, es la centralidad de ciertos temas lo que permite diferenciar los ámbitos prioritarios para su calidad de vida.

TABLA 2.

Número de citas asociadas a cada dimensión

\begin{tabular}{|c|c|c|c|}
\hline \multicolumn{2}{|c|}{ Hombres semivalentes } & \multicolumn{2}{|c|}{ Mujeres semivalentes } \\
\hline Dimensión & Citas & Dimensión & Citas \\
\hline Economía & 30 & Familia & 18 \\
\hline Salud & 28 & Salud & 16 \\
\hline Pareja & 17 & Economía & 12 \\
\hline Familia & 14 & Amigos/vecinos & 12 \\
\hline $\begin{array}{l}\text { Amigos/ } \\
\text { vecinos }\end{array}$ & 12 & Residencia/barrio & 12 \\
\hline $\begin{array}{l}\text { Actividades no } \\
\text { cotidianas }\end{array}$ & 10 & Futuro & 10 \\
\hline $\begin{array}{l}\text { Integración } \\
\text { Social }\end{array}$ & 10 & Rutina & 10 \\
\hline $\begin{array}{l}\text { Residencia/ } \\
\text { barrio }\end{array}$ & 8 & Integración Social & 8 \\
\hline Rutina & 5 & $\begin{array}{l}\text { Actividades no } \\
\text { cotidianas }\end{array}$ & 3 \\
\hline Trabajo & 4 & Soledad & 3 \\
\hline $\begin{array}{l}\text { Preocupación } \\
\text { Social }\end{array}$ & 3 & Trabajo & 2 \\
\hline
\end{tabular}

De acuerdo a la Tabla $N^{0} 2$, en los discursos de los hombres sobresale la dimensión economía y en el de las mujeres aparecen mayores contenidos asociados a la dimensión familia, mientras que para ambos la dimensión salud resulta gravitante. Estas dimensiones operan como articuladores de las otras dimensiones reconocidas, pues suelen actuar como inhibidoras o potenciadoras de los demás ámbitos. Así por ejemplo, el contar con más dinero (dimensión economía) permitirá acceder a mejores servicios, o realizar actividades con amigos; tener una buena salud (dimensión salud), facilitará el realizar 
actividades y utilizar los espacios del barrio y la ciudad; etc. Es por ello que para mostrar los resultados obtenidos en las entrevistas nos centraremos en las dimensiones familia, economía y salud. Luego en la discusión se irán incluyendo otras dimensiones conforme vaya siendo relevante.

Tanto para hombres como para mujeres adultas mayores semivalentes, la dimensión economía es una de las más significativas. Para ambos, los recursos económicos resultan cruciales para poder seguir llevando su vida cotidiana, sosteniendo cierto grado de autonomía y autovalencia, apareciendo éstas últimas como la aspiración subyacente en los discursos. Sin embargo, la gran diferencia radica en que el envejecimiento, según relatan los hombres, ha significado una merma muy importante en los ingresos. En tanto en las mujeres, si bien declaran tener más gastos, pues su grado de dependencia así lo requiere, no siempre refieren en sus discursos a la disminución de ingresos. Ello se puede entender a partir de una valoración diferenciada de esta dimensión (economía), producto de la construcción de identidades de género en base a las cuales fueron y son socializados.

La merma en los ingresos de los hombres se debería básicamente a la jubilación, a las bajas pensiones y a su condición de semivalencia que genera situaciones en que son los hijos o hijas quienes administran sus bienes, provocando en ellos un sentimiento de diminución de control económico:

"Con \$300 (mil) me conformaría. Yo fui siempre comisionista, incluso no tenia sueldo, pero las comisiones eran altas. Pero con lo que le dije, usted dirá: este caballero tiene los bolsillos rotos acumulando billetes. No veo nada, tengo que pedirles por favor que me pasen plata para cortarme el pelo, después de 50 años de trabajo (...) Así es la vida. Aquí se pagan 250 mil de arriendo y tengo tres, cuatro casas. Ellos (mis hijos) cobran los arriendos. Esa es mi jubilación, limosnero, que bonito" (Gustavo, 83 años, NSE alto).

Los recursos económicos son en general tematizados, sobre todo en los hombres, como facilitadores en la consecución de deseos y necesidades. Esto significa que la disminución de los ingresos impactaría en el sentimiento de autoeficacia, cuestionando en gran medida uno de los elementos centrales de la identidad masculina tradicional -el de proveedor eficaz-, arrojándolos en una de las dimensiones de lo que Thumala, Arnold, Urquiza, Blanco y Vogel, (2009) han descrito como la exclusión.

La familia es una dimensión también relevante en la evaluación de la calidad de vida de las mujeres. Esta situación puede ser vinculada a varias cuestiones. De las mujeres entrevistadas, sólo una vivía sola, mientras que las otras vivían con familiares (hijos, hijas, nietos, nietas). Los hombres, por otra parte, vivían con sus parejas, excepto uno que vivía con familiares. Es así como en el caso de los hombres, la dimensión pareja está más presente en los discursos que la dimensión familia. Este tipo de distribución del tipo de residencia no fue intencionado en el muestreo, lo que podría estar poniendo en evidencia el hecho de que las mujeres son más longevas que los hombres, y por lo mismo sufren mayormente la viudez ${ }^{3}$. Para la comprensión de esta dimensión resulta relevante, por lo tanto, observarla desde una perspectiva de género, en cuanto el cruce vejez y género nos muestra que los hombres al enviudar tienden a volver a casarse y por lo tanto, vivir con su nueva cónyuge a diferencia de las mujeres que

\footnotetext{
3 Según los datos de la encuesta del programa Domeyko Envejecimiento, un $32 \%$ de la muestra eran viudos y viudas, pero al separarlos por género $14,6 \%$ correspondía a hombres y $39,1 \%$ a mujeres.
} 
viven la viudez en familia con hijos e hijas, quienes asumirán su cuidado cuando estas lo requieran, debido a la pérdida de autovalencia y autonomía, generando dependencia. Dicha situación implica que las mujeres adultas mayores semivalentes, se sienten muy agradecidas y a la vez culpables por la carga que significan al interior de sus familias:

"Aquí, mi hija no me deja mojarme las manos porque el agua es muy helada, entonces ¿donde es mejor?... con mi hija" (Felicia, 88 años, NSE medio).

En el caso de los hombres mayores, reconocen la importancia que sus parejas 0 esposas tienen en sus vidas, siendo consideradas siempre como un apoyo fundamental en su situación de semivalencia, pero también son valoradas por la compañía y el cariño que les proporcionan, compartiendo la cotidianeidad lo cual resulta esencial dada la poca movilidad que la mayoría de los entrevistados presenta. Por lo mismo, aquellos hombres que se encuentran solos consideran que sería importante para sentirse satisfechos con su vida el contar con una pareja. Pese a ello, descartan la posibilidad de realizar esto debido a que dicen estar acostumbrados a la soledad.

No obstante las diferencias en el tipo de residencia, el papel que desempeña la familia en el caso de las mujeres responde al rol asignado por la división del trabajo, orientada por las diferencias de género, según la cual, los hijos e hijas son responsabilidad principal de las mujeres, lo que en la vejez pareciera implicar que lo afectivo estaría vinculado mayormente al vínculo filial, lo que se refleja en los discursos:

"Entonces, cuando ellos me llaman yo soy la mujer más dichosa, pero cuando no ando preocupada, que será de ellos, me gustaría verlos" (Claudia, 69 años, NSE bajo).
Dentro de la dimensión familia, las relaciones positivas son interesantes por el modo en que influyen en la calidad de vida de las personas mayores. Más allá de que se reconozca el beneficio que dichas relaciones tienen sobre la calidad de vida propia, es el ver a los hijos y nietos bien lo que les hace sentir bien. En otras palabras, la calidad de vida propia es una consecuencia de la posibilidad de que los hijos y nietos sean felices. Así, la satisfacción con la vida, sobre todo entre quienes viven con familiares que son sus cuidadores, resulta de la satisfacción que dicha familia pudiera experimentar. Esto se condice con la dependencia de la que hablábamos antes, es decir, la relación sujeto cuidado-sujeto cuidador resulta ser altamente dependiente para ambos miembros de la díada, generando este tipo de proyecciones.

Cabe destacar que, conforme avanza la edad, los vínculos familiares se van verticalizando, es decir, los pares hermanos, primos- van envejeciendo y/o muriendo, por lo que hijos/as, sobrinos/as, nietos/as, se constituyen en la red familiar más cercana (Gastrón en Anigstein, 2010). Las hijas $e$ hijos son vistos como proveedores de ayuda, y las personas mayores que no tuvieron hijos sienten esta falta de alguien que los hubiese podido apoyar durante su vejez y en su situación particular de semivalencia.

En cuanto a la salud se refiere, tanto en hombres como mujeres resulta ser otra de las dimensiones que más destacan en los discursos. La salud es relatada desde la perspectiva de la pérdida, es decir, se tematiza en la medida que significa molestias y desventajas, sobre todo vinculadas a la autonomía y autovalencia.

En algunos casos, las situaciones adversas de salud realzan una sensación de incertidumbre y a su vez dan lugar a la valoración de la vida, desde el momento 
que se cree que la muerte es muy posible y cercana. Es decir, la salud como dimensión invita a las personas entrevistadas a reflexionar sobre los efectos negativos que ha tenido y tiene la vejez y su condición de semivalencia, pero a la vez les permite identificarse como envejecientes, desde un sentimiento de estar vivos que les genera satisfacción:

"Entonces por eso mismo no tengo ningún miedo de morirme o que tenga... puede ser mañana como un año, o a lo mejor en 10 años, no sé, depende del caballero de arriba" (Pablo, 80 años, NSE alto).

Pero no solamente se mencionan las enfermedades que se tienen y cómo repercuten en su bienestar, sino también se hace referencia a la calidad de la atención médica recibida, especialmente al trato que se les da en los centros de salud.

Las evaluaciones sobre el bienestar actual varían: algunos son negativos sobre su estado de salud, y otros aceptan su condición aunque reconocen las limitaciones que les trae. Es interesante ver que aunque físicamente las personas pueden tener problemas en su movilidad, la evaluación de su estado de salud es más positiva cuando se refieren a sus capacidades mentales, específicamente la memoria.

Junto a estos resultados presentados a nivel descriptivo, emerge la posibilidad de construir un diagrama interpretativo sobre cómo se comportan las dimensiones identificadas. En el diagrama $\mathrm{N}^{0} 1$ se integran de manera dinámica las dimensiones descritas que compondrían la calidad de vida de las personas mayores semivalentes, con el fin de observar y comprender la interacción entre ellas.

- Endo-entorno: se compone del contexto y las relaciones del propio
El diagrama fue construido a través de un proceso inductivo, en que las dimensiones identificadas en relación a la calidad de vida fueron ordenadas de acuerdo a su funcionalidad, es decir, cómo se manifiestan y como operan en el contexto de la calidad de vida de las personas mayores semivalentes. La síntesis conceptual que presentamos a continuación podríamos decir que se inscribe en un marco constructivista, en el sentido de que indicamos dimensiones diferenciales de la calidad de vida que en su interacción nos permitirían comprender como ésta es entendida por las personas mayores.

Se han distinguido, por una parte, condiciones y por otra, entornos. Las condiciones, corresponden a aquellas dimensiones que están influyendo en la calidad de vida de las personas mayores, y estas pueden ser vividas como heterodeterminadas $0 \quad$ como autoderminadas. Las primeras, corresponden a condiciones vividas como externas que posibilitarían o inhibirían la calidad de vida de las personas mayores, determinando cuáles serían los elementos del entorno que podrán experimentar y de qué modo. Las personas experimentarían este nivel, como una menor posibilidad de decisión sobre su manifestación. Por su parte, las condiciones autodeterminadas corresponderían a la experiencia cotidiana, más abierta a las posibilidades de incidencia por parte del sujeto.

Los entornos, corresponden al contexto donde se manifiesta y experimenta la calidad de vida, siendo aquí donde puede ser observada bajo la influencia de las condiciones. No deben ser confundidos con espacios físicos, aunque los incorporan. Los entornos involucran el vínculo relacional de las personas mayores y la sociedad. Se han distinguidos tres niveles de manifestación de la calidad de vida:

sujeto y su núcleo social directo. Es el ámbito de lo personal, familiar, 
biográfico. Es el contexto de construcción de su identidad individual, la subjetividad, en sí y para sí. Su nivel es micro.

- Meso-entorno: contexto de relaciones intermedias. Es el ámbito de los vínculos y relaciones sociales $y$ contexto de relaciones intermedias, interpersonales y de redes sociales. Es el puente del nivel micro al macro, y del nivel macro al micro.
- Exo-entorno: contexto de la identidad de la persona mayor en tanto sujeto social. Es el ámbito de lo sociocultural. Es el contexto donde se juega la inclusión/exclusión social, 10 normativo y de la construcción de la persona en cuanto sujeto productor/producto de lo social. Su nivel es macro.

DIAGRAMA 1.

Condiciones y entornos para la comprensión de la calidad de vida en personas mayores

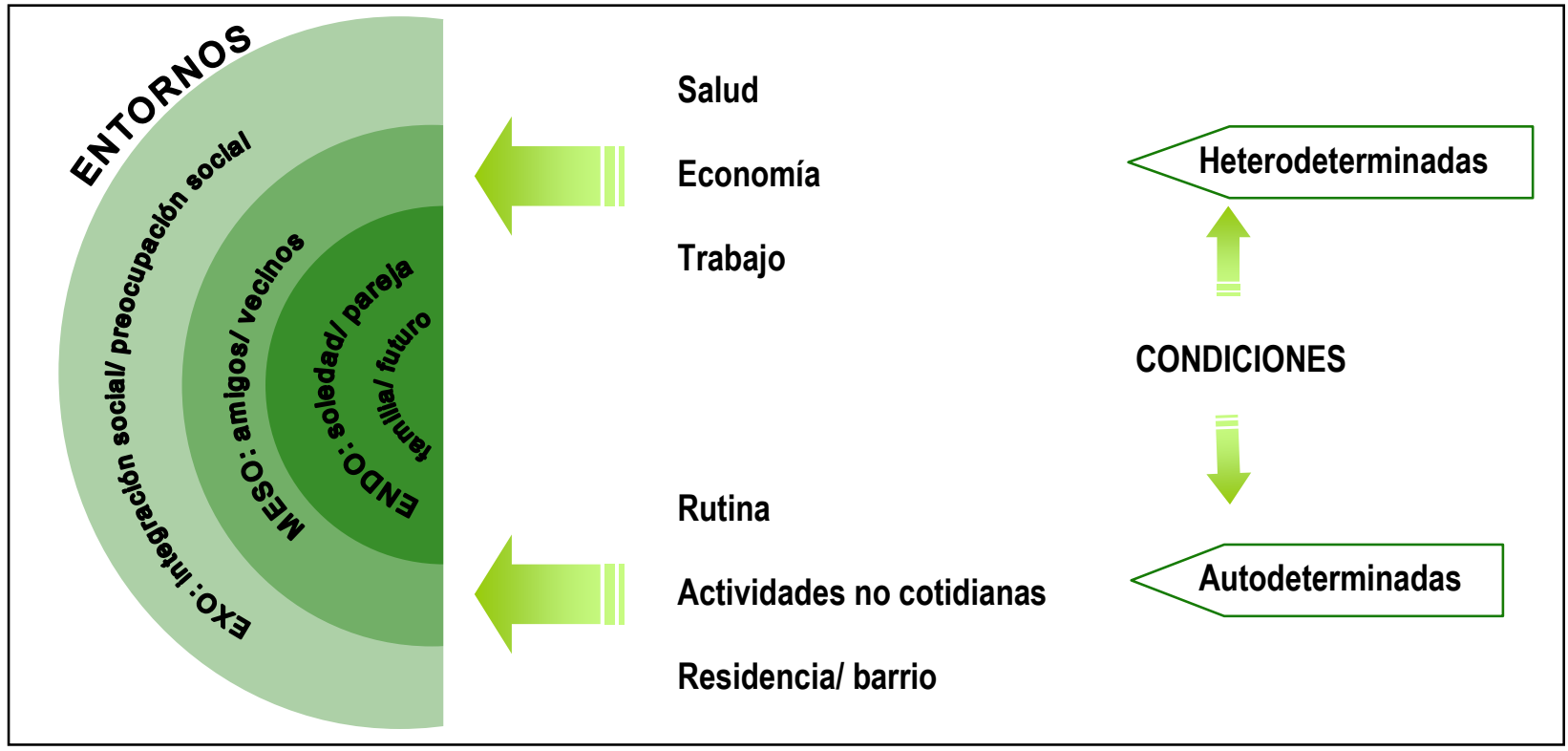

Las personas mayores que participaron de ésta investigación experimentan la economía, la salud y el trabajo como condiciones heterodeterminadas. En cuanto a la primera de éstas, se vincula a temas como contar con dinero para necesidades básicas y recreación, para costear emergencias, etc. La economía, al igual que la salud, se constituye en elemento vinculante, que se relaciona si no con todos, con la gran mayoría de los entornos con los cuales interactúan las personas mayores semivalentes y a los cuales hacen referencia al hablar sobre la calidad de vida. Especialmente se alude a razones económicas al no poder acceder a "darse un gusto" -que correspondería a un endo-entorno- 0 a tener más posibilidad de realizar actividades recreativas -mesoentorno. También se reconocen las diferencias por el estrato socioeconómico al cual se pertenece, no sólo por poder optar a una mayor pensión -de manifestación a nivel de exo-entorno-, sino también porque biográficamente se indica que las personas mayores de nivel socioeconómico más alto han envejecido de mejor manera, encontrándose mejor física y anímicamente, esta última, realidad que se manifiesta en el endo-entorno. 
La Tabla $\mathrm{N}^{0} 3$ da cuenta de la relevancia que asume la dimensión economía en el caso de los varones, identificándola como una condición heterodeterminada, pues al cruzarla con su incidencia en la calidad de vida, ésta escapa a las posibilidades de decidir sobre cómo los afecta dicha condición. Aquellos adultos mayores semivalentes que cuentan con una pensión más alta 0 con ingresos complementarios por arriendo de propiedades -exo-entorno-, se muestran molestos debido a que muchas veces no son ellos quienes están a cargo del manejo de su dinero, sino sus hijos, vale decir, en un endo-entorno.

TABLA 3.

Número de citas asociadas a cada dimensión

\begin{tabular}{|c|c|c|c|c|c|c|}
\hline \multirow[b]{2}{*}{ Condición } & \multicolumn{3}{|c|}{ Hombres semivalentes } & \multicolumn{3}{|c|}{ Mujeres semivalentes } \\
\hline & Dimensión & Citas & Total & Dimensión & Citas & Total \\
\hline \multirow{3}{*}{ Heterodeterminada } & Economía & 30 & \multirow{3}{*}{62} & Salud & 16 & \multirow{3}{*}{30} \\
\hline & Salud & 28 & & Economía & 12 & \\
\hline & Trabajo & 4 & & Trabajo & 2 & \\
\hline \multirow{3}{*}{ Autodeterminada } & Actividades no cotidianas & 10 & \multirow{3}{*}{23} & Residencia/ barrio & 12 & \multirow{3}{*}{25} \\
\hline & Residencia/ barrio & 8 & & Rutina & 10 & \\
\hline & Rutina & 5 & & $\begin{array}{l}\text { Actividades no } \\
\text { cotidianas }\end{array}$ & 3 & \\
\hline
\end{tabular}

La economía, que a un nivel de exo-entorno puede mostrarse favorable, al vivenciarse en un nivel de endo-entorno, hace que se relativice su evaluación e incidencia en la calidad de vida. Igualmente, si se aspira que esta condición sea experimentada como autodeterminada, pero se mantiene $y$ manifiesta con características de heterodeterminación, puede hacer la vivencia de esta condición negativa en un nivel de endo-entorno.

Al igual que la economía, la salud es una de las condiciones con mayor relevancia en los discursos, lo que muestra la centralidad que tiene en la evaluación de la calidad de vida. En el grupo de las personas mayores semivalentes, su grado de dependencia y las enfermedades que presentan, constituyen un impedimento para realizar actividades cotidianas y recreativas a nivel de endoentorno, repercutiendo principalmente en las relaciones con su meso-entorno (amigos, vecinos, barrio) y familia. Asimismo, representa un cambio en sus posibilidades de autonomía, pues requieren ser atendidos por un cuidador. Esto último, posiciona a la salud como una condición heterodeterminada, pues el quiebre de la posibilidad de autonomía hace imposible la autodeterminación.

Las personas mayores valoran las actividades recreativas para su calidad de vida, principalmente porque les permiten mantenerse activos. Estas actividades las realizan tanto dentro como fuera de la casa, dependiendo de las posibilidades que tienen debido a su condición de semivalencia. Muchas veces esto implica que actividades que tradicionalmente han servido como vía para mantener determinadas redes sociales, vínculos con amigos y conocidos comienzan a abandonarse, y como consecuencia, repercute en sus relaciones personales. En este punto, la calidad de vida se vería afectada negativamente desde el mesoentorno, comenzado paulatinamente a perder los vínculos con personas que no pertenecen a sus familias en las llamadas actividades no cotidianas (participar de un club, asistir a un taller 0 a reuniones con amigos, caminar, entre otras) y que tanto hombres como mujeres valoran bastante dentro de las condiciones autodeterminadas que inciden en su calidad de vida. 
A diferencia de estas actividades no cotidianas vinculadas al entretenimiento $y$ recreación, las actividades rutinarias que ellos mismo realizan, o apoyan o tan solo observan (si cuentan con un cuidador), no son consideradas como positivas para la calidad de vida, pues se las significa como una concatenación de acontecimientos que marca el paso del tiempo, sin que tenga una relevancia o que sea un aporte para ellos. A pesar de ser una condición autodeterminada, las actividades de la vida diaria 0 rutinarias, pasan a ser heterodeterminadas, donde las personas mayores semivalentes pierden su manejo y control, pasando a ser externas y a experimentarse como una condición que depende de un tercero, ya sea el cuidador/a o un familiar cercano.

Más allá de las relaciones específicas con amigos, vecinos y familiares, las personas mayores mencionan como una dimensión que afecta su bienestar y calidad de vida las posibilidades de integración que existen en la sociedad. Esto es, que sus opiniones y necesidades sean consideradas, se les valore como persona, que se de solución a los problemas específicos de las personas mayores, y que en un nivel de meso y exoentorno, la sociedad no los excluya producto de su edad o situación de semivalencia.

\section{DISCUSIÓN Y CONCLUSIONES}

Si bien la calidad de vida tiene que ver tanto con condiciones objetivas -externas a los sujetos- como con percepciones subjetivas -desde los sujetos mismos-, puede resultar igualmente reduccionista polarizar su comprensión. Comprender la calidad de vida desde una perspectiva dinámica, nos lleva a una epistemología constructivista más integral y no atomística para aproximarnos al concepto. La complejidad del término no sólo da cuenta de un problema conceptual, sino que también de la realidad misma de la calidad de vida de las personas. Esta hace referencia a la comprensión de los sujetos en cuanto seres integrales que experimentan su vida en un determinado contexto, ya sea relacional, físico o sociocultural, y en un momento histórico y biográfico particular. Es así como los resultados de esta investigación dan cuenta de que si bien las condiciones más relevadas para la calidad de vida pueden ser las mismas, su valoración y formas de manifestación presentan particularidades de género y grados de autovalencia, relativizando su significado y relación con la calidad de vida.

En el caso de las personas mayores semivalentes, las condiciones pueden ser entendidas como la vivencia de mayor 0 menor autonomía y autovalencia frente a la vida, en la que ciertos aspectos de ésta, son más o menos posibles de ser incididos en base a las propias decisiones. Si bien la auto 0 heterodeterminación no depende necesariamente de la edad de las personas o la autovalencia de éstas, lo que interesa es el significado que la pérdida de autovalencia adquiere en la relación de las personas mayores con sus condiciones de calidad de vida.

Al observar las particularidades de género de la condiciones señaladas como más relevantes (economía, familia y salud), nos damos cuenta que para los hombres fue más significativo lo vinculado a la economía y para las mujeres lo relacionado con la familia -lo que podría deberse a la distribución tradicional de los roles de género- en ambos se hacen presentes éstos aspectos, al igual que la salud. Dichas condiciones pueden ser experimentadas por las personas mayores participantes como heterodeterminadas 0 autoderminadas, 10 que va a influir en la mirada negativa 0 positiva que se tenga de la calidad de vida. La posibilidad de que las condiciones sean vividas como autodeterminadas es muy gravitante cuando se evalúa positivamente la calidad de vida y viceversa, cuando se las vive como heterodeterminadas. En este sentido, la vivencia que se tiene de lo que 
hemos llamado condiciones en el caso de directamente relacionada con la salud, ya que esta introduce una variación en la forma en que otras condiciones son experimentadas, pudiendo cambiar su nivel de determinación (generalmente a heterodeterminación) y con ello, la percepción de calidad de vida de las personas mayores (Diagrama $\mathrm{N}^{\circ} 2$ ). Por las personas mayores semivalentes estaría ejemplo, una condición tan autodeterminada como las actividades de la vida diaria, puede transformarse en heterodeterminada al acrecentarse la dependencia de un cuidador, influyendo directamente en la percepción de la calidad de vida de ese sujeto.

DIAGRAMA 2.

Cambios en la determinación de las condiciones al manifestarse en los entornos para las personas mayores semivalentes.

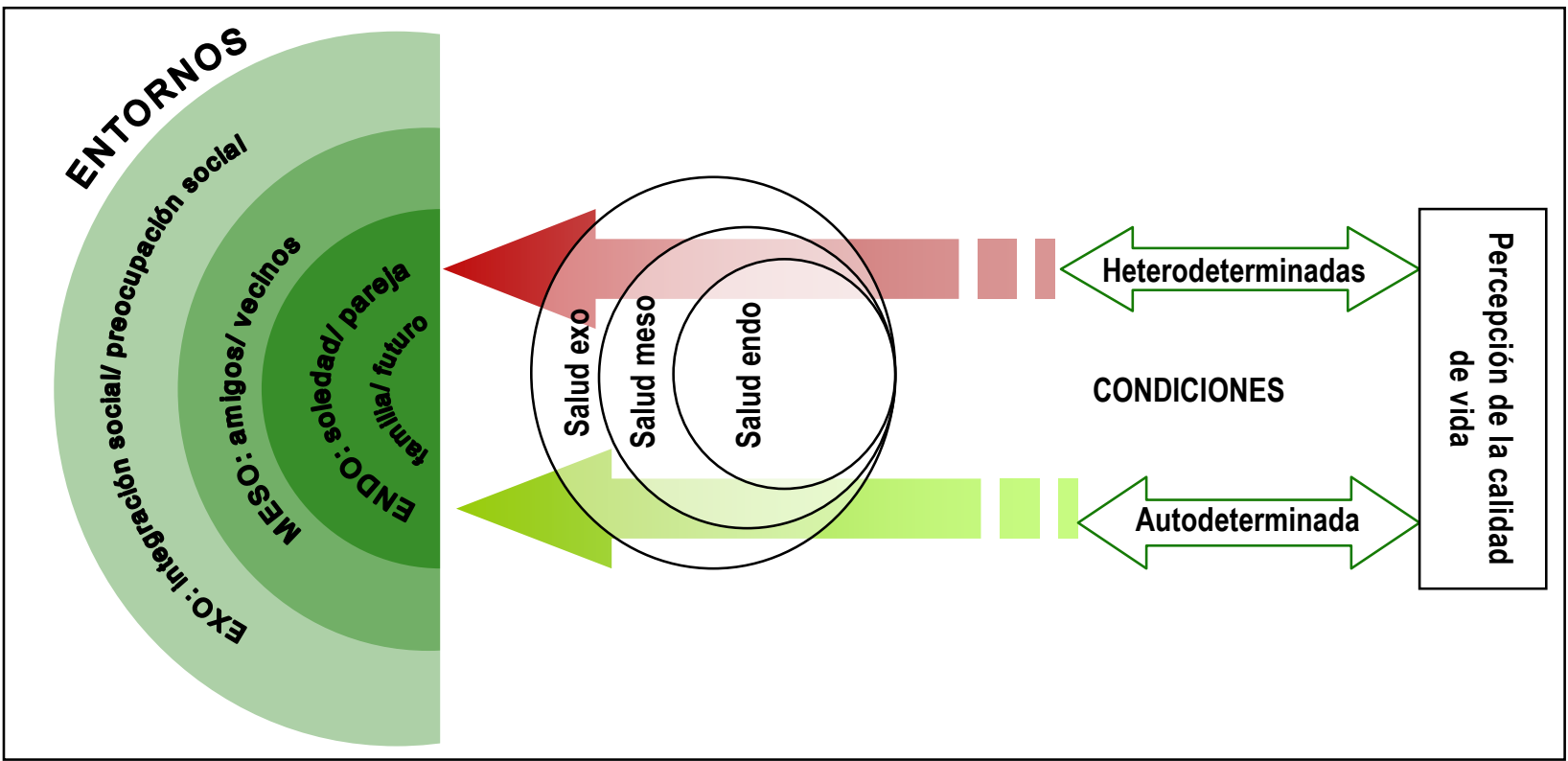

La vivencia de las condiciones tiene lugar en lo que se ha llamado entornos: endo-entorno, meso-entorno y exo-entorno. La experiencia de las condiciones puede variar según el entorno en el que tenga lugar, y así como influirse mutuamente, por ejemplo, la vivencia de dependencia en el endo-entorno, podría provocar cierto retraimiento en el meso y exoentonos.

Mediante este diagrama interpretativo, hemos arribado a algunas conclusiones relevantes para esta población en particular, que logran esbozar las claves desde la que estarían evaluando su calidad de vida las personas mayores.

La evaluación de la calidad de vida en personas mayores semivalentes, estaría dependiendo de la experiencia de las condiciones como heterodeterminadas, mas su evaluación sería positiva 0 negativa dependiendo de cómo la gestión de los entornos resulta en algún grado de autodeterminación. La autodeterminación para las personas mayores semivalentes es una eje central en la evaluación de su calidad de vida, de igual forma como lo planteado por Anigstein (2010) para las mujeres mayores frágiles, son la autonomía y autovalencia.

En la descripción de los aspectos más relevantes, se relata la desventaja en la que se sienten respecto de su situación económica, la relación de dependencia con sus familias y la pérdida creciente de la salud. Estas evaluaciones indican una vivencia mucho más cercana a la heterodeterminación, lo que se ve acentuado con la alta valoración de la autonomía, en todos los aspectos que aparece subyaciendo los discursos. 
Los entornos, constituyen en este diagrama, los escenarios donde las trayectorias van teniendo lugar, y que facilitan o dificultan la vivencia de las condiciones. A modo de ejemplo, un endo-entorno amigable $y$ estimulante, le permitirá al adulto mayor semivalente, sentirse más confiado para desempeñarse en sus otros entornos, pudiendo tal vez sobrellevar la vivencia poco afortunada de alguna de las condiciones. Así, el diagrama aquí planteado permite la coexistencia de evaluaciones aparentemente contradictorias sobre diversas condiciones de la calidad de vida. Esto debido a que permite recoger analíticamente los discursos de vida de las personas mayores, manteniendo a la vez su cohesión, tal como plantea Mcadams (2005) la agencia humana permite seleccionar del menú de experiencias posibles en sus vidas, construyéndose así historias de vida que incluso pueden desafiar al statu quo, pero a la vez se encuentra con los sentimientos y necesidades del individuo, en la medida que este intenta satisfacer un nicho psicológico.

Este diagrama interpretativo permite comprender en alguna medida la interrelación presente entre los distintos factores que confluyen en la evaluación de la calidad de vida y el rol fundamental que ostenta la vivencia que se tiene de algunos de éstos, en determinados escenarios, y específicamente el papel de la vivencia de autodeterminación de la propia vida, que resulta ser central en la evaluación de la calidad de vida. Esto último plantea claramente la necesidad de idear y confeccionar estrategias que permitan fortalecer la vivencia de autodeterminación frente a las diferentes condiciones y en los distintos entornos en que los adultos mayores semivalentes transitan.

Lo aquí planteado es esbozado en el Informe de la Segunda Asamblea Mundial del Envejecimiento (Madrid, 2002), el cual plantea el desafío que el cambio demográfico significa para la sociedad, ya que hace necesario aumentar las oportunidades para las personas $-y$ especialmente para las personas de edadcon el fin de aprovechar al máximo sus capacidades de participar en todos los aspectos de la vida. Por ello, los mecanismos de inclusión y la consecuente integración se vuelven cruciales, cuando se piensa en la calidad de vida de las personas mayores semivalentes. Generar enfoques que permitan trabajar con la complejidad del concepto de calidad de vida, así como las particularidades de cada segmento de la población resulta esencial para incorporar las necesidades de la población mayor a la agenda pública de los gobiernos y a las prioridades que las sociedades establecen, reconociendo al mismo tiempo los componentes estructurales y subjetivos de la calidad de vida, así como su interacción.

Al analizar la calidad de vida de las personas mayores semivalentes desde una perspectiva cualitativa y de las experiencias y significados individuales y sociales, observamos que las dimensiones de ésta no son absolutas. Así, el diagrama interpretativo propuesto busca incorporar el dinamismo del concepto, asumiendo que la calidad de vida, no es o positiva o negativa, bueno o mala, sino que su percepción dependerá desde qué condición se la evalúe y en qué entorno se la experimente. Asimismo, asume la necesidad planteada por varios autores (Aranibar, 2001; Fernández, 1997; George, 2006; Lawton en Barros et al., 2004) de conceptualizar la calidad de vida desde una perspectiva multidimensional, considerando aspectos subjetivos de las personas, a la vez que aquellos que son objetivos y/o externos, de manera dinámica, incorporando particularidades de la población o grupo en cuestión y definiendo algunos elementos y relaciones más genéricas o nomotéticas. Es decir, el diagrama propuesto ha sido elaborado a partir del análisis de las propias percepciones de las personas mayores semivalentes, y por tanto, el modo en que los entornos y condiciones han sido 
presentado pueden variar al ser aplicado a otras poblaciones y contextos.

\section{REFERENCIAS}

Andrews, F.M. and Withey, S.B. (1974) Developing Measures of Perceived Life Quality: Results from Several National Surveys, Social Indicators Research 1, 1-26.

Anigstein, M.S (2010) Observaciones De Mujeres Adultas Mayores Frágiles Sobre Su Calidad De Vida En La Ciudad De Santiago De Chile.Tesis para optar al grado de Magíster en Antropología y Desarrollo. Departamento de Antropología, Facultad de Ciencias Sociales, Universidad de Chile.

Aranibar, P. (2001) Acercamiento conceptual a la situación del adulto mayor en América Latina. Cepal, Santiago de Chile.

Barros, C., Avendaño, C. y Forttes, A. (2004). Componentes de la calidad de vida del adulto mayor y factores asociados. Informe de Investigación Proyecto FONDECYT 1020643. Santiago de Chile.

Berjano, E. (2005) Dependencia y calidad de vida en los mayores. Rev. Mult. Gerontol. 2005; 15(3): 144-154.

CELADE (2006) Manual sobre indicadores de calidad de vida en la vejez. Santiago de Chile, diciembre de 2006.

Dijkers, M. (2007) "What's in a name?" The indiscriminate use of the "Quality of life" label, and the need to bring about clarity in conceptualizations. International journal of nursing studies 2007;44 (1):153-5.

Elkinton J.R. (1966) Medicine and the quality of life. Ann Intern Med. 1966;

64(3): 711-714.

Fernández Ballesteros, R. (1997) Calidad de vida en la vejez: condiciones diferenciales. Anuario de Psicología 1997, 73. Barcelona: Universitat de Barcelona. 89-104. Extraído el 8 de marzo de 2010 desde http://www.raco.cat/index.php/An uarioPsicologia/article/viewFile/61355/8 8779

George, L. (2006) Perceived Quality of Life. En Binstock and George Ed. Handbook of Aging and the Social Sciences. Sixth Edition. London, Inglaterra: Academic Press-Elsevier

Hidalgo Rasmussen, C. (2008) De los Comportamientos de riesgo a la Calidad de vida de los Adolescentes. Guadalajara, México: Ed. Universidad de Guadalajara.

Mcadams, D.P. (2005) Studying Lives In Time: A Narrative Approach en R. Lévy, P. Ghisletta, J. Le Goff, D. Spini, \& E. Widmer, Towards an Interdisciplinary Perspective on the Life Course. Advances in Life Course Research (Vol. X). Elsevier Ltd.

MIDEPLAN. (2009) Encuesta de Caracterización Socioeconómica Nacional Casen 2006. Resultados Discapacidad. Santiago de Chile: MIDEPLAN. Extraído el 16 de marzo de 2011 desde http://www.mideplan.gob.cl/casen2009/ Discapacidad_casen_2009.pdf

MINSAL. (2006). Adultos Mayores en control, según edad y sexo, por condición delestado funcional, Diciembre 2006. Departamento de Estadísticas e información deSalud. Santiago de Chile: MINSAL. Extraído el 27 de abril de 2009 desde http://163.247.51.54/rem2006/pobl_dic/ adulto_mayor_s6.php?tipomes=12\&tip oano $=2006$

Moons P, Budts W, De Geest S. (2006) "Critique on the conceptualisation of quality of life: A review and evaluation of different conceptual approaches".International Journal of Nursing Studies, 43 (7): 891-901.

OMS (2001) Clasificación Internacional del Funcionamiento, de la Discapacidad y de la Salud (CIF). OMS, 2001 http://www.sidar.org/recur/desdi/pau/cif. php 
Naciones Unidas (2002) Plan Internacional de Acción de Madrid sobre el Envejecimiento Naciones Unidas, Nueva York. http://www.imsersomayores.csic.es/doc umentos/documentos/onu-informe01.pdf

Osorio, P (2006) "La longevidad más allá de la biología. Aspectos socioculturales", en Papeles del CEIC, $n^{0}$ 22, CEIC (Centro de Estudios sobre la Identidad Colectiva), Universidad del País Vasco, http://www.ehu.es/CEIC/pdf/22.pdf

Osorio, P., Torrejón M.J. y Vogel N. (2008) "Aproximación a un concepto de calidad de vida en la vejez. Escuchando a las personas mayores". Revista de Psicología Vol. XVII (1): 101-108.

Pedrero, E. (2001) La calidad de vida y las personas mayores. Monografía presentada a los seminarios de psicogerontología. Extraído el 2 de Julio de 2009 desde http://psiconet.com/tiempo/monografias /calidad.htm

Puts, S., Widdershoven, Heldens, Lips y Deeg. (2007, Marzo). What does quality of life mean to older frail and non frail community dwelling adults in the Netherlands? Quality of Life Research [Revista Electrónica] 16, 263-277. Obtenido de la base de datos Springer el 25 de abril de 2009 desde http://www.springerlink.com/content/u6 5550221g670836/

Schalock R.L. (1999) Hacia una nueva concepción de la discapacidad. En: Verdugo MA, Borja $F$ y Jordan de Urries (coord.). Hacia una nueva concepción de la discapacidad. (pp 80108). Salamanca: Amaru 1999.
Schwartzmann, L. (2003). Calidad de Vida relacionada con la salud: Aspectos conceptuales. Revista Ciencia y Enfermería IX (2): 9-21.

Taylor, S.J. y Bogdan R. (1987) Introducción a los métodos cualitativos de investigación: La búsqueda de significados". Editorial Paidós Básica. 1987 de todas las ediciones en castellano. pp. 100-132

Thumala, D., Arnold, M., Urquiza, A., Blanco, C., Vogel, N. (2009). Inclusión y exclusión social del adulto mayor en Chile. Opiniones, expectativas y evaluaciones de la población chilena sobre diferentes modalidades de inclusión y exclusión social de las personas adultas mayores. Programa de estudios sistémicos. Envejecimiento y Vejez en Chile. Facso-Pulso. Universidad de Chile. Extraído el 6 de abril de 2010 desde http://www.esistemicosvejez.cl/upload/e st_pdf/7.pdf

Vera, M. (2007) Significado de la calidad de vida del adulto mayor para sí mismo y para su familia. Anales de la Facultad de Medicina [en línea] 2007, 68 [fecha de consulta: 31 de agosto de 2010] Disponible en: $<$ http://redalyc.uaemex.mx/redalyc/src/i nicio/ArtPdfRed.jsp?iCve=37968312> ISSN 1025-5583. 